\title{
ON PERFECT SUMMABILITY OF DOUBLE SEQUENCES
}

\author{
J. D. HILL
}

1. Introduction. The purpose of the present note is to point out that the results of Banach on perfect summability of simple sequences $^{1}$ may be extended to certain cases of double sequence summability. The main result obtained is embodied in the theorem of $\S 3$.

It will be convenient to begin with the following definitions and notations. We denote by $c$ the class of all real double sequences $x \equiv\left\{\xi_{k l}\right\}$ for which the principal limit $\lim _{k, l} \xi_{k l} \equiv \xi$ exists finite; by $r c$, the subclass of $c$ for which the row limits $\lim _{l} \xi_{k l} \equiv \xi_{k}^{r},(k=0,1,2, \cdots)$ and the column limits $\lim _{k} \xi_{k l} \equiv \xi_{l}^{c},(l=0,1,2, \cdots)$, exist finite; and by $r c r n$, the subclass of $r c$ throughout which $\xi_{k}^{r}=\xi_{l}^{c}=\xi=0$, $(k, l=0,1,2, \cdots)$. With the conventional definitions of addition and multiplication by a constant the classes $r c$ and $r c r n$ are linear spaces, and they become Banach spaces upon introduction of the norm $\|x\| \equiv \sup _{k, l}\left|\xi_{k l}\right|$.

Let $A \equiv\left(a_{i j k l}\right)$ be a given infinite matrix of real numbers. We shall be concerned with transformations of the form

$$
A_{i j}(x) \equiv \sum_{k, l} a_{i j k l} \xi_{k l}, \quad i, j=0,1,2, \cdots,
$$

on the elements $x \equiv\left\{\xi_{k l}\right\}$ of $r c$. More precisely, if $A_{i j}(x),(i, j$ $=0,1,2, \cdots)$, exists for every $x \varepsilon r c$ and if the corresponding sequence $\left\{A_{i j}(x)\right\}$ belongs to $r c[c]$, one says that $r c$ is transformed into $r c[c]$ by the method of summability corresponding to the matrix $A$, or simply, by the method $A$. If $A(x) \equiv \lim _{i, j} A_{i j}(x)=\xi$ for every $x \varepsilon r c$, the method is called regular. A regular transformation of $r c$ into itself is called completely regular if it is also regular by rows and columns, that is to say, if $A_{i}^{r}(x) \equiv \lim _{j} A_{i j}(x)=\xi_{i}^{r},(i=0,1,2, \cdots)$, and $A_{j}^{c}(x) \lim _{i} A_{i j}(x)=\xi_{j}^{c},(j=0,1,2, \cdots)$, for every $x \varepsilon r c$.

For later reference we recall that the condition

$$
\sup _{i, j} \sum_{k, l}\left|a_{i j k l}\right| \equiv M<\infty
$$

is necessary ${ }^{2}$ in order that $A$ transform $r c r n$ into $r c$.

If the system of equations

$$
\sum_{k, l} a_{i j k l} \xi_{k l}=\eta_{i j}, \quad i, j=0,1,2, \cdots,
$$

${ }^{1}$ Banach, Théorie des Opérations Linéaires, pp. 90-95.

${ }^{2}$ See Hamilton, Transformations of multiple sequences, Duke Mathematical Journal, vol. 2 (1936), pp. 29-60; in particular, p. 42, \#5. 
has a unique solution $\left\{\xi_{k l}\right\}$ (belonging to $r c$ or not) corresponding to each $\left\{\eta_{i j}\right\} \& r c$, the method $A$ will be called reversible. ${ }^{3}$

REMARK. If $A$ is reversible, it can be shown ${ }^{4}$ that there exist linear functionals $f_{k l}(y)$ defined in $r c$, such that for each $y \equiv\left\{\eta_{i j}\right\} \varepsilon r c$ we have $\sum_{k, l} a_{i j k l} f_{k l}(y)=\eta_{i j},(i, j=0,1,2, \cdots)$. This fact is essential to the proof of Lemma 4 in $\$ 3$.

$A$ will be said to be of type $M$ if the conditions ${ }^{5}$

$$
\sum_{i, j}\left|\alpha_{i j}\right|<\infty, \quad \sum_{i, j} \alpha_{i j} a_{i j k l}=0, \quad k, l=0,1,2, \cdots,
$$

always imply $\alpha_{i j}=0,(i, j=0,1,2, \cdots)$.

Any method $A$ which is simultaneously reversible, completely regular, and of type $M$ will be called perfect. ${ }^{6}$

Frequent use will be made of the following particular double sequences: the sequence $X$, all of whose terms are 1 ; the sequence $X_{k}^{r}$, $(k=0,1,2, \cdots)$, all of whose terms are 0 except those in the $k$ th row which are 1 ; the sequences $X_{l}^{c},(l=0,1,2, \cdots)$, all of whose terms are 0 except those in the $l$ th column which are 1 ; and the sequences $X_{k l},(k, l=0,1,2, \cdots)$, all of whose terms are 0 except for the term common to the $k$ th row and $l$ th column, which is 1 .

Corresponding to any given $x \varepsilon r c$ we set

$$
\begin{aligned}
x_{i} \equiv \xi X & +\sum_{k=0}^{p_{i}}\left(\xi_{k}^{r}-\xi\right) X_{k}^{r}+\sum_{l=0}^{p_{i}}\left(\xi_{l}^{c}-\xi\right) X_{l}^{c}+\sum_{k, l=0}^{p_{i}}\left(\xi-\xi_{k l}\right) X_{k l} \\
& +\sum_{k, l=0}^{p_{i}, q_{i}}\left(\xi_{k l}-\xi_{k}^{r}\right) X_{k l}+\sum_{k, l=0}^{q_{i}, p_{i}}\left(\xi_{k l}-\xi_{l}^{c}\right) X_{k l}, \quad i=0,1,2, \cdots .
\end{aligned}
$$

Then each $x_{i} \varepsilon r c$, and it is easy to verify that $p_{i}, q_{i},\left(q_{i} \geqq p_{i} \rightarrow \infty\right)$, can be so chosen that $\lim _{i} x_{i}=x$.

2. The general form of linear functionals in $r c$. The following fact will be needed in the proof of Lemma 1 in $\$ 3$.

Every linear functional $f(x)$ defined in $r c$ is of the form

$$
f(x)=C \xi+\sum_{k} C_{k}^{r} \xi_{k}^{r}+\sum_{l} C_{l}^{c} \xi_{l}^{c}+\sum_{k, l} C_{k l} \xi_{k l}
$$

and

${ }^{3}$ Compare with Banach, op. cit., p. 90.

${ }^{4}$ See Banach, op. cit., p. 47 , Theorem 10 . The proof given is easily extended to the system (1.3).

- Compare with Banach, op. cit., p. 90.

- Compare with Banach, op. cit.. D. 90. 


$$
\|f\|=|C|+\sum_{k}\left|C_{k}^{r}\right|+\sum_{l}\left|C_{l}^{c}\right|+\sum_{k, l}\left|C_{k l}\right| \text {. }
$$

Proof. Let $f(x)$ be an arbitrary linear functional defined in $r c$, and set $f(X) \equiv A, f\left(X_{k}^{r}\right) \equiv A_{k}^{r}, f\left(X_{l}^{c}\right) \equiv A_{l}^{c}, f\left(X_{k l}\right) \equiv A_{k l},(k, l=0,1,2, \cdots)$. We show first that each of the series $\sum_{k}\left|A_{k}^{r}\right|, \sum_{l}\left|A_{l}^{c}\right|, \sum_{k, l}\left|A_{k l}\right|$ is convergent.

Let $x$ \& $r c r n$ be arbitrary and set $x_{i j} \equiv \sum_{k, l=0}^{i, j} \xi_{k l} X_{k l}, \quad(i, j=0,1$, $2, \cdots)$. Then each $x_{i j} \varepsilon$ rcrn and since $x_{i j} \rightarrow x$ as $i, j \rightarrow \infty$, it follows that $f\left(x_{i j}\right)=\sum_{k, l=0}^{i, j} \xi_{k l} A_{k l}$ tends to a limit, namely $f(x)$, as $i, j \rightarrow \infty$. In order that this be true for every $x \varepsilon$ rcrn it is necessary ${ }^{7}$ that $\sum_{k, l}\left|A_{k l}\right|<\infty$.

On the other hand let $\left\{\xi_{k}\right\}$ be an arbitrary null sequence, and let $x \equiv\left\{\xi_{k l}\right\}$ be such that for each fixed $k=0,1,2, \cdots$ we have $\xi_{k l}=\xi_{k}$ for $l=0,1,2, \cdots$. If we set $x_{i} \equiv \sum_{k=0}^{i} \xi_{k} X_{k}^{r},(i=0,1,2, \cdots)$, then each $x_{i} \varepsilon r c$ and $x_{i} \rightarrow x$ as $i \rightarrow \infty$. Since $f\left(x_{i}\right)=\sum_{k=0}^{i} \xi_{k} A_{k}^{r}$, it follows that the series $\sum_{k} \xi_{k} A_{k}^{r}$ is convergent for every null sequence $\left\{\xi_{k}\right\}$. Consequently, the condition $\sum_{k}\left|A_{k}^{r}\right|<\infty$ must be satisfied, ${ }^{8}$ and it is evident that the symmetrical condition $\sum_{l}\left|A_{l}^{c}\right|<\infty$ must also hold.

Now let $x \varepsilon r c$ be chosen arbitrarily, and let $x_{i}$ be defined as in (1.5) so that $x_{i} \rightarrow x$. Then $f\left(x_{i}\right) \rightarrow f(x)$, and from (1.5) we find that

$$
\begin{aligned}
f(x)= & \xi A+\sum_{k}\left(\xi_{k}^{r}-\xi\right) A_{k}^{r}+\sum_{l}\left(\xi_{l}^{c}-\xi\right) A_{l}^{c} \\
& +\sum_{k, l}\left(\xi+\xi_{k l}-\xi_{k}^{r}-\xi_{l}^{c}\right) A_{k l} .
\end{aligned}
$$

On rearranging the latter expression and setting

$$
\begin{array}{rlrl}
C & \equiv A-\sum_{k} A_{l b}^{r}-\sum_{l} A_{l}^{c}+\sum_{k, l} A_{k l}, & C_{k}^{r} \equiv A_{k}^{r}-\sum_{l} A_{k l}, \\
C_{l}^{c} \equiv A_{l}^{c}-\sum_{k} A_{k l}, & C_{k l} \equiv A_{k l},
\end{array}
$$

$f(x)$ reduces to the form (2.1) as was to be shown.

Regarding the evaluation of $\|f\|$, it is clear that $\|f\|$ does not exceed the value given in (2.2). The converse statement can be readily shown to follow from the relation $\left|f\left(x_{n}\right)\right| \leqq\|f\|$, wherein $x_{n} \equiv\left\{\xi_{k l}^{n}\right\}$ for each $n=0,1,2, \cdots$ is defined as follows: ${ }^{9} \xi_{k l}^{n} \equiv \operatorname{sgn} C_{k l}$ for $0 \leqq k, l \leqq n$; $\equiv \operatorname{sgn} C_{k}^{r}$ for $0 \leqq k \leqq n, n<l<\infty$; $\equiv \operatorname{sgn} C_{l}^{c}$ for $0 \leqq l \leqq n, n<k<\infty$;

${ }^{7}$ See Hamilton, loc. cit., p. 41, \#1.

${ }^{8}$ See Banach, op. cit., p. 86. Merely replace $(c)$ by $\left(c_{0}\right)$ in the discussion at the top of the page.

จ To insure $\left\|x_{n}\right\|=1$ for every $n$, let $\operatorname{sgn} \alpha \equiv 1$. if $\alpha \geqq 0$; $\equiv-1$ if $\alpha<0$. 
$\equiv \operatorname{sgn} C$ for $n<k, l<\infty$. Inasmuch as we make no use of (2.2), the remaining details may be omitted.

3. The analogues of Banach's results. We come next to the following four lemmas. Since these bear a direct analogy to those of Banach, both in statement and method of proof, we shall give here only the proof of the first lemma. The second one is a straightforward corollary of the first, and the reader who is familiar with the original lemmas will encounter no difficulty in supplying the remaining two proofs. ${ }^{10}$

Lemma 1. Let $A$ be a completely regular method and let $y_{0} \equiv\left\{\eta_{i j}^{0}\right\}$ be an arbitrary element of rc. If the conditions (1.4) always imply $\sum_{i, j} \alpha_{i j} \eta_{i j}^{0}=0$, then corresponding to each $\epsilon>0$ there exists an $x \varepsilon$ rc such that

$$
\left|A_{i j}(x)-\eta_{i j}^{0}\right|<\epsilon, \quad i, j=0,1,2, \cdots .
$$

Proof. Let $G$ denote the subset of $r c$ of all sequences of the form $\left\{\eta_{i j}\right\} \equiv\left\{A_{i j}(x)\right\}$ for $x$ ranging over $r c$. Evidently $G$ is a linear set.

Let us assume that for some particular $\epsilon>0$, the condition (3.1) cannot be satisfied. This is equivalent to assuming that $y_{0}$ is not a limit element of $G$. In such a case there must exist a linear functional $F(y)$ defined in $r c$ such that ${ }^{11} F\left(y_{0}\right)=1$ and $F(y)=0$ for every $y \varepsilon G$. In view of $\$ 2$ we are therefore led to relations of the following sort:

$$
C_{\eta}^{0}+\sum_{i} C_{i}^{r} \eta_{i}^{0 r}+\sum_{j} C_{j \eta_{j}^{0 c}}^{c}+\sum_{i, j} C_{i j}{ }^{0} \eta_{i j}=1
$$

$$
C \eta+\sum_{i} C_{i}^{r} \eta_{i}^{r}+\sum_{j} C_{j \eta_{j}^{c}}^{c}+\sum_{i, j} C_{i j} \eta_{i j}=0 \text { for all }\left\{\eta_{i j}\right\}_{\varepsilon} G
$$

wherein $\sum_{i, j}\left|C_{i j}\right|<\infty$. From the definition of $G$ and the fact that $A$ is completely regular, (3.3) may be written

$$
C \xi+\sum_{i} C_{i}^{r} \xi_{i}^{r}+\sum_{j} C_{j}^{c} \xi_{j}^{c}+\sum_{i, j} C_{i j} A_{i j}(x)=0 \text { for all } x \varepsilon r c
$$

Now by virtue of (1.2) we have $\sum_{i, j, k, l=0}^{n}\left|C_{i j} a_{i j k l} \xi_{k l}\right| \leqq M \cdot\|x\|$ $\cdot \sum_{i, j}\left|C_{i j}\right|<\infty$ for each $n=0,1,2, \cdots$ and each $x \equiv\left\{\xi_{k l}\right\} \varepsilon r c$. Consequently, (3.4) may be expressed in the form

$$
\Phi(x) \equiv C \xi+\sum_{i} C_{i}^{r} \xi_{i}^{r}+\sum_{j} C_{j}^{c} \xi_{j}^{c}+\sum_{k, l} \xi_{k l} \sum_{i, j} C_{i j} a_{i j k l}=0,
$$

valid for all $x \varepsilon r c$. Allowing $x$ to take in turn the values $X_{k l}, X_{k}^{r}, X_{l}^{c}$,

${ }^{10}$ In this connection, however, see the remark in $\$ 1$.

11 Banach, op. cit., p. 57, \$3, Lemma. 
$X,(k, l=0,1,2, \cdots)$, in $\Phi(x)$, we find successively the following relations:

$$
\sum_{i, j} C_{i j} a_{i j k l}=0, \quad k, l=0,1,2, \cdots,
$$

and $C_{i}^{r}=C_{j}^{c}=C=0,(i, j=0,1,2, \cdots)$. Equation (3.2) then reduces to $\sum_{i, j} C_{i j} \eta_{i j}^{0}=1$, and this in view of (3.5) contradicts the hypothesis. The proof is therefore complete.

LEMMA 2. If the method $A$ is completely regular and of type $M$, then corresponding to each $\left\{\eta_{i j}^{0}\right\} \varepsilon r c$ and each $\epsilon>0$, there exists an $x \varepsilon r c$ satisfying condition (3.1).

LEMMA 3. If $x_{0}$ is a bounded sequence transformed into rc by a completely regular method $A$, then corresponding to each $\epsilon>0$ there exists an $x$ \& $r c$ such that

$$
\left|A_{i j}(x)-A_{i j}\left(x_{0}\right)\right|<\epsilon, \quad i, j=0,1,2, \cdots .
$$

LEMMA 4. Let $x_{0}$ be any sequence transformed into rc by a completely regular and reversible method $A$. If corresponding to each $\epsilon>0$ there exists an $x \varepsilon r c$ satisfying (3.6), then the sequence $x_{0}$ is summable to the same principal limit by every method $B$ which transforms $r c$ into $c$ regularly and which transforms into $c$ every sequence that $A$ transforms into $r c$. Moreover, if $B$ is completely regular and transforms into rc every sequence that $A$ does, then the row and column limits assigned by $B$ to $x_{0}$ will be respectively equal to those assigned by $A$.

Finally, the preceding lemmas may be combined to yield the following counterpart of Banach's theorems.

THEOREM. Let the method $A$ be perfect [completely regular and reversible], and let the method $B$ transform rc into $c$ regularly and transform into $c$ every sequence that $A$ transforms into rc. Then every sequence [bounded sequence] $x_{0}$ transformed into $r c$ by $A$ is transformed into $c$ by $B$ with the same principal limit. Moreover, if $B$ is completely regular and transforms into rc every sequence that $A$ does, then the row and column limits assigned by $B$ to $x_{0}$ will be respectively equal to those assigned by $A$.

Michigan State College 